the compound and obtain pyrimidine from it. In this respect the pyrimidine analog differs from the pyridine analog studied earlier; ${ }^{2}$ the latter compound was about as effective a source of pyrimidine as thiamine.

1 The terms pyrimidine and thiazole are used in this paper to refer to the intermediates of thiamine, 4-methyl-5- $\beta$-hydroxyethyl thiazole and 2-methyl-5-bromomethyl-6-aminopyrimidine hydrobromide.

${ }^{2}$ Robbins, W. J., Proc. Nat. Acad. Sci. 27, 419 (1941).

\title{
ON THE PHYSICAL CHARACTERISTICS OF THE PERSEUS CLUSTER OF NEBULAE
}

\section{By F. Zwicky}

Norman Bridge laboratory of Physics, California Institute of Technology

Communicated July 31,1942

A. The Radial Distribution of Nebulae in the Perseus Cluster.-In a previous paper ${ }^{1}$ counts of nebulae in the field which covers the Perseus cluster were communicated. These counts refer only to nebulae which can be distinguished on limiting exposures taken with the 18-inch Schmidt telescope on Palomar Mountain. As was done previously ${ }^{2}$ with the data on the clusters in Coma and in Hydra we here compare the distribution of the nebulae in the Perseus cluster with the distribution which Emden ${ }^{3,4}$ deduced theoretically for the bounded isothermal gravitational gas sphere. In table 1 are listed the average numbers $N_{r}$ per square degree of the nebulae brighter than about the absolute photographic magnitude $M_{p}=-14.3$ as a function of the distance $r$ from the center of the Perseus cluster. From the numbers $N_{r}$ previously ${ }^{1}$ given, the numbers $N_{r}$ listed here are obtained by subtracting 3.0 nebulae per square degree which represent the average background of the field nebulae in which the Perseus cluster appears imbedded. The reduction of the observed distribution to the standard Emden curve of the projected densities $D$ of the isothermal gas sphere is accomplished by plotting in figure 1 the values of $5.5 N_{r}$ as a function of the associated Emden radius $r_{1}$ which is related to the actual radius vector by the equation

$$
r=\alpha r_{1}
$$

A good fit between the observational data and the theoretical curve is obtained if the structural length (or structural index) ( $^{5} \alpha$ is set equal to $10 / 3$ minutes of arc or, in absolute measure, if we take 11 million parsecs as the distance of the Perseus cluster

$$
\alpha=3.30 \times 10^{22} \mathrm{~cm} .
$$


In figure 1 the drawn-out curve represents the radial distribution ${ }^{4}$ of the projected density $D-37 / 1000$ of that bounded isothermal Emden sphere which is obtained from the density $D$ of the infinite sphere by subtracting the constant $37 / 1000$. Although this constant must be expected to vary from cluster to cluster it is seen from figure 1 that the adoption of the value

TABLE 1

Radial Distribution of Nebulae in the Perseus Cluster

\begin{tabular}{|c|c|c|c|c|c|}
\hline $\begin{array}{l}\text { IN MINUTES } \\
\text { OF ARC P }\end{array}$ & $\begin{array}{l}\quad N_{r} \\
\text { PER SQUARE DEGREE }\end{array}$ & $5.5 N_{r}$ & $r_{1}$ & $1000 D$ & $1000 D-37$ \\
\hline 5 & 554 & 3047 & 1.5 & 2430 & 2393 \\
\hline 10 & 214 & 1177 & 3 & 1524 & 1487 \\
\hline 15 & 145 & 798 & 4.5 & 968 & 931 \\
\hline 20 & 96 & 528 & 6 & 649 & 612 \\
\hline 25 & 59 & 325 & 7.5 & 476 & 439 \\
\hline 30 & 52 & 286 & 9 & 366 & 329 \\
\hline 35 & 51 & 281 & 10.5 & 305 & 268 \\
\hline 40 & 34 & 187 & 12 & 254 & 217 \\
\hline 45 & 22 & 121 & 13.5 & .. & . \\
\hline 50 & 21.4 & 117 & 15 & 195 & 158 \\
\hline 60 & 16.0 & 88 & 18 & 157 & 120 \\
\hline 70 & 15.7 & 86.4 & 21 & $\cdots$ & . \\
\hline 80 & 10.8 & 59.4 & 24 & . & $\cdots$ \\
\hline 90 & 12.0 & 66.0 & 27 & . & . \\
\hline 100 & 10.4 & 57.2 & 30 & 92.0 & 55 \\
\hline 110 & 5.3 & 29.2 & 33 & $\cdots$ & $\cdots$ \\
\hline 120 & 8.0 & 44.0 & 36 & . & $\cdots$ \\
\hline 130 & 7.7 & 42.4 & 39 & $\cdots$ & . \\
\hline 140 & 5.2 & 28.6 & 42 & . & $\cdots$ \\
\hline 150 & 4.6 & 25.3 & 45 & 63.1 & 26.1 \\
\hline 160 & 4.1 & 22.6 & 48 & $\cdots$ & $\ldots$ \\
\hline 170 & 1.22 & 6.7 & 51 & $\cdots$ & $\cdots$ \\
\hline 180 & 2.30 & 12.7 & 54 & . & $\ldots$ \\
\hline 190 & 2.33 & 12.8 & 57 & $\cdots$ & $\because$ \\
\hline 200 & 1.27 & 7.0 & 60 & 49 & 12.0 \\
\hline 210 & 1.40 & 7.7 & 63 & . & $\cdots$ \\
\hline 220 & 2.93 & 16.1 & 66 & $\cdots$ & $\cdots$ \\
\hline 230 & 5.25 & 28.9 & 69 & . & . \\
\hline 240 & 0.95 & 5.2 & 72 & $\cdots$ & $\cdots$ \\
\hline 250 & 1.73 & 9.4 & 75 & 39 & 2.0 \\
\hline
\end{tabular}

$37 / 1000$ used already in the analysis of the clusters in Coma and in Hydra results in an equally satisfactory representation of the nebular counts in the Perseus cluster. Because of the logarithmic scale used in figure 1 for $N_{r}$, the fluctuations, which are proportional to $\left(N_{r} / r\right)^{1 / 2}$ appear inordinately large for small values of $N_{r}$. The absolute values of the fluctuations are, however, within the theoretically expected range. Like the clusters in Coma and in Hydra, the Perseus cluster may consequently be considered as 
a large scale assembly of nebulae which is statistically stationary. Basing our further considerations on this result we may again, as in the case of the clusters in Coma and in Hydra, carry through the quantitative analysis described elsewhere. ${ }^{2}$ Proceeding in this manner we expect to arrive at a correct prediction for the magnitude of the velocity dispersion in the Perseus cluster in dependence of its structural length $\alpha$ and its central density $\rho_{0}$.

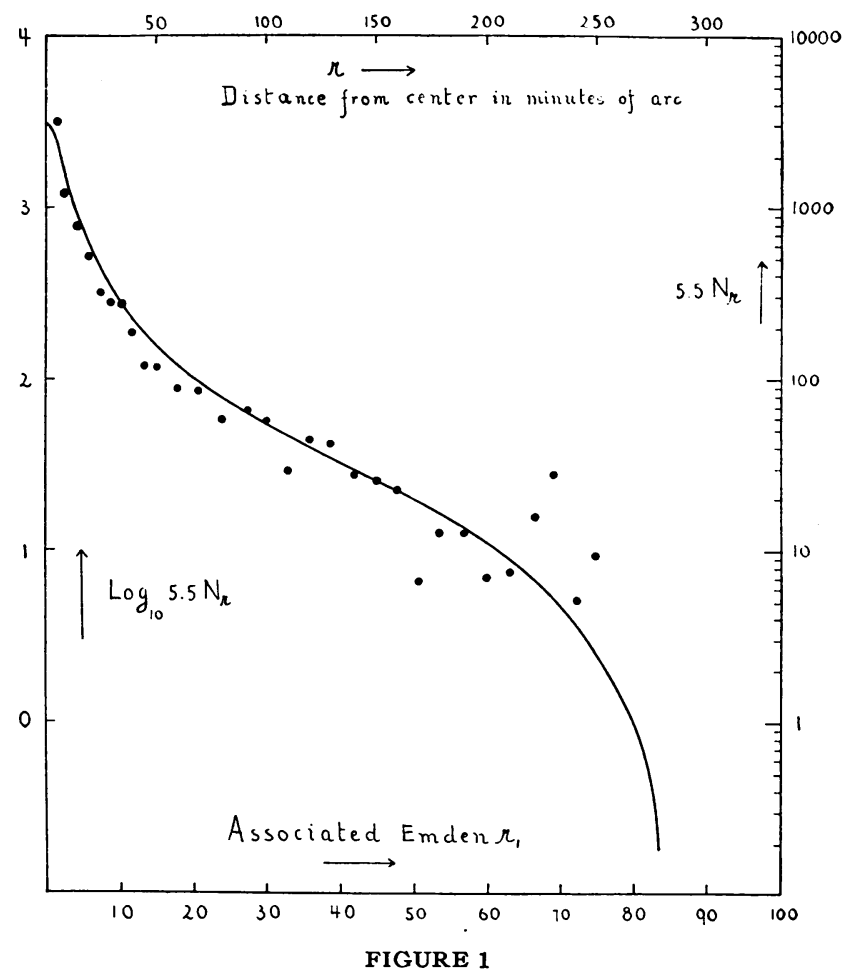

B. Some Physical Characteristics of the Perseus Cluster.-In the Emden isothermal sphere the projected central density $\sigma_{0}$ and the real space density $\rho_{0}$ are connected by the equation ${ }^{2}$

$$
\rho_{0}=\sigma_{0} / 3.03 \alpha .
$$

Choosing the megaparsec as the unit of length, introducing $\alpha=10 / 3$ minutes of arc $=1 / 18$ degree and $\sigma_{0}=3030 / 5.5$ nebulae per square degree, and finally converting angular measures into megaparsecs we obtain for the center of the cluster the density

$$
P_{0}=460,000 \text { nebulae per cubic megaparsec. }
$$

This number includes only the nebulae listed in table 1 which are all brighter 
than about the absolute photographic magnitude $M_{p}=-14.3$. We denote with the average mass of these nebulae and we obtain for the real average central density of matter per $\mathrm{cm}^{3}$

$$
\rho_{0}=4.6 \times 10^{5} \mathrm{\gamma} \overline{\mathrm{IH}} / 3 \times 10^{73}=1.53 \times 10^{-67} \mathrm{\gamma} \overline{\mathrm{AH}},
$$

where $\gamma$ is of the order of unity when it is assumed that the actual central density of matter is not materially greater than that incorporated in the brighter nebulae here considered. . As was shown before ${ }^{5}$ the velocity dispersion $(\bar{w})^{1 / 2}$ can be calculated from the relation

$$
\left(\bar{w}^{2}\right)^{1 / 2}=\alpha\left[12 \pi \Gamma \rho_{0}\right]^{1 / 2}
$$

where $\Gamma$ is the universal gravitational constant. The following table 2 gives $\rho_{0}$ and $\gamma \bar{A}$ in dependence of various assumed values for the velocity dispersion $\left(\bar{w}^{2}\right)^{1 / 2}$. The mass of the sun is ${ }_{\odot}=2 \times 10^{33} \mathrm{~g}$.

TABLE 2

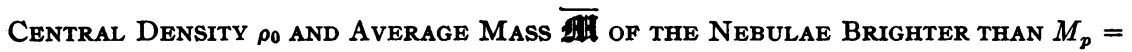
- 14.3 in the Perseus Cluster in Dependence of the Velocity Dispersion

$\left(\bar{w}^{2}\right)^{1 / 2}$
IN KM./sEC.
250
500
750
1000
1250
1500

$$
\begin{aligned}
& \text { po IN G./CM. } \\
& 2.2 \times 10^{-25} \\
& 8.8 \times 10^{-25} \\
& 2.0 \times 10^{-24} \\
& 3.5 \times 10^{-24} \\
& 5.5 \times 10^{-24} \\
& 7.9 \times 10^{-24}
\end{aligned}
$$

$\gamma \overline{24} / 24$

$7.1 \times 10^{\circ}$

$2.8 \times 10^{10}$

$6.4 \times 10^{10}$

$1.1 \times 10^{11}$

$1.8 \times 10^{11}$

$2.6 \times 10^{11}$

According to information kindly supplied me by Dr. Hubble the data on the radial velocities $w_{r}$ of nebulae in the Perseus cluster are still very scant. An estimate on the basis of these data indicates that $\left(\bar{w}_{r}^{2}\right)^{1 / 2} \cong 350-400$ $\mathrm{km}$./sec. which results in

$$
\left(\bar{w}^{2}\right)^{1 / 2}=\left(3 \bar{w}_{r}^{2}\right)^{1 / 2} \cong 600-700 \mathrm{~km} . / \mathrm{sec} .
$$

Unless faint nebulae or dark matter contribute far more to the mass of the Perseus cluster than the nebulae which are brighter than $M_{p}=-14.3$ the . average mass of these nebulae becomes of the order of $5 \times 10^{10}$ though we cannot at the present time check this conclusion directly we may apply the following quantitative test to the interpretation given here of the observations on the Perseus cluster by comparing these observations with those made on the Coma cluster. If we denote with the indices $P$ and $C$ the quantities which refer to the clusters in Perseus and in Coma, respectively, we have according to (6)

$$
\left(\bar{w}^{2}\right)_{P}=\left[\alpha_{P}^{2} \rho_{0 P} / \alpha_{C}^{2} \rho_{O C}\right]\left(\bar{w}^{2}\right)_{C} .
$$


Inserting the numerical values for $\alpha_{P} / \alpha_{C}$ and $\rho_{0 P} / \rho_{0 C}$ it follows that

$$
\left(\bar{w}^{2}\right)^{1 / 2} \text { Persei }<0.62\left(\bar{w}^{2}\right)^{1 / 2} \text { Comao, }
$$

where the "smaller" sign means "somewhat smaller" since the ratio $\rho_{0 P} / \rho_{0 C}$ was set equal to the ratio per unit cube of the nebulae brighter than the absolute magnitudes $M_{p}=-14.3$ and $M_{p}=-14.5$ for the clusters in Perseus and in Coma, respectively. Adopting $1100 \mathrm{~km}$./sec. for the dispersion in radial velocities in the Coma cluster this leads to

$$
\left(\bar{w}_{r}^{2}\right)^{1 / 2} \text { Persei }=\text { somewhat smaller than } 680 \mathrm{~km} . / \mathrm{sec} .
$$

for the predicted dispersion in the radial velocities of the nebulae in the Perseus cluster, a result which compares favorably with the dispersion of $400 \mathrm{~km}$./sec. derived by Hubble as a very rough estimate from a few directly measured velocities.

Distance in $10^{6}$ light years

Diameter in $10^{6}$ light years

Diameter in minutes of arc

Total number of nebulae brighter than photog. mag. $m_{L}$

Limiting photog. mag. $m_{L}$

$m_{\max }$. = photog. mag. of brightest nebula

$M_{\max .}=$ estimated abso-

lute photog. mag. of brightest nebula

$M_{L}=M_{\max }+\left(m_{L}-\right.$

$m_{\text {max. }}$.

$\alpha=$ structural index

Number of nebulae per cubic megaparsec in center of cluster with $M \leqslant M_{L}$
TABLE 3

$\begin{array}{ccc}\begin{array}{c}\text { PHYSICAL CHARACTERISTICS OF THE CLUSTHRS OF NEBULAE IN } \\ \text { GYORA }\end{array} \\ \begin{array}{c}\text { OERSEUS } \\ 45.0\end{array} & 23.8 & 35.9 \\ 4.4 & 4.7 & 6.0 \\ 340 & 680 & 566 \\ & & \\ 670 & 270 & 360 \\ 16.6 & 16.2 & 16.5 \\ 14.1 & 13.1 & 13.8 \\ & & \\ -17.0 & -17.0 & -17.0 \\ & & \\ -14.5 & -13.9^{*} & -14.3 \\ 2^{\prime}= & 4^{\prime}= & 3.33^{\prime}= \\ 48 \times 10^{22} \mathrm{~cm} . & 2.56 \times 10^{22} \mathrm{~cm} . & 3.30 \times 10^{22} \mathrm{~cm} .\end{array}$

$2,100,000$

880,000

460,000

* In a previous paper ${ }^{2} M_{L}$ for the Hydra cluster by mistake was given as -13.1 instead of -13.7 as would have followed from the considerations there used.

C. Review of Some of the Physical Characteristics of the Clusters of Nebulae in Coma, in Hydra and in Perseus.-The clusters in Coma, in Hydra and in Perseus are the three largest among the spherically symmetrical clusters which are in reach of the 18-inch Schmidt telescope on Palomar Mountain. It was shown in a series of investigations ${ }^{2}$ that the observed radial distribution curves of the brighter nebulae in these clusters can all be reduced to the 
same standard curve which represents the radial distribution of the density of matter in a bounded isothermal gravitational gas sphere. The two reduction factors involved in the reduction of the observational data to the theoretical curve can be expressed in terms of the velocity dispersion in the cluster, its central density or the average mass and number per unit volume of the nebulae involved. The substitution of observed values for the quantities mentioned into the theoretical relations results in a satisfactory check of these relations and furnishes significant support for the following contentions: (1) clusters of nebulae represent statistically stationary distributions of matter as far as the brighter nebulae are concerned; (2) Newton's law of gravitation as first good approximation satisfactorily represents the interactions of nebulae separated by distances of the order of one million light years; and (3) the masses of the brighter nebulae are of the order of $10^{10} \mathrm{AH}_{\odot}$ to $10^{11} \mathrm{MH}_{\odot}$.

In table 3 are listed some of the data which are significant in the analysis of the large-scale physical constitution of the three clusters of nebulae in Coma, in Hydra and in Perseus.

The diameters for the three clusters given in table 3 refer of course only to those distances from the center of the clusters at which the emergence of the brighter cluster nebulae from the background nebulae can be statistically ascertained. When sufficient observations with more powerful telescopes are available so that the fainter member nebulae of the clusters can also be included in the counts it will probably be found that the diameters of the clusters are still larger than those given in table 3 .

Unfortunately only a few more spherically symmetrical clusters can be reached with the 18-inch Schmidt telescope and these are considerably less rich in nebulae than those listed in table 3. The important question of the segregation of nebulae of different brightness can also only partly be solved until larger Schmidt telescopes are available. In addition, the velocity distribution as a function of the brightness of the nebulae must be investigated. The problems of the segregation of nebulae and of the dispersion in the velocities demand also further theoretical clarification since the classical statistical mechanics gives no answer to the fundamental questions which arise in connection with gravitational coöperative assemblies which are composed of such diverse elements as the nebulae, the stars and the constituents of intergalactic and interstellar matter. The uniformity of the structure of symmetrical clusters of nebulae observed so far and the quantitative agreement of their observed physical characteristics with those derived theoretically for the isothermal gravitational "gas" sphere suggest that the short-time scale associated customarily with the hypothesis of an expanding universe will perhaps become the less attractive the more the investigations on the large-scale distribution of matter in the universe progress. 
${ }^{1}$ Zwicky, F., Proc. Nat. Acad. Sci., 28, 317-320 (1942).

${ }^{2}$ Zwicky, F., Ibid., 28, 150 (1942); Astrophys. Jour., 95, 555 (1942).

${ }^{3}$ Emden, R., Gaskugeln, Teubner, Leipzig, 1907.

${ }^{4}$ Hubble, E., Astrophys. Jour., 71, 231 (1930).

${ }^{5}$ Zwicky, F., Th. von Kármán Anniversary Volume, May, 1941, p. 137.

\section{THE EPIDEMIC CURVE}

\section{By Edwin B. Wilson and Mary H. Burke}

\section{Harvard School of Public Health}

Communicated July 15, 1942

In 1929 Soper $^{1}$ developed a theory of the epidemic curve based on tracing the rise and fall of the disease by "generations" of successive groups of infectious cases. If in the $i$ th generation there are $C_{i}$ infectious cases and $S_{i}$ susceptibles and if there be a constant number $A$ of susceptibles per infectious generation coming into the population, his equations to determine the number of cases and the number of susceptibles in the next generation were $^{2}$

$$
\frac{C_{i+1}}{C_{i}}=\frac{S_{i}}{m},
$$

and

$$
S_{i+1}=S_{i}-C_{i+1}+A,
$$

where $m$ is the number of susceptibles necessary for one old case to produce just one new case. In the stationary endemic condition $C_{i+1}=C_{i}$, $S_{i}=m, S_{i+1}=S_{i}$ and $C_{i+1}=A$. The length of the generation was taken to be the "incubation period." Thus if a fortnight be taken as the incubation period for measles and if there are 150 children per fortnight coming into the population through birth and growing up (with due allowance for deaths and emigration and immigration) measles could maintain itself in a stationary endemic condition with 150 cases per fortnight, the quantity $m$ would have to be determined by enumerating the susceptibles in the population under such conditions, and might well be of the order of 4 to 5 years' worth of recruits $A$, which at 150 per fortnight would be 15,600 to 19,500 . As measles does not occur in a steady endemic condition but in sharp epidemics, it is necessary according to Soper's equations that $m$ should be variable or that there should be an accumulation of susceptibles to a number considerably greater than $m$ before the epidemic, with an alternative deficit of susceptibles considerably below $m$ after the epidemic.

Already in February, 1928, Dr. Wade H. Frost when delivering the Cutter Lectures in Preventive Medicine at the Harvard Medical School 